\title{
THE FLUX AND SPECTRAL VARIABILITY OF NGC6814 AS OBSERVED
} WITH EXOSAT

\author{
J.P.D. MITTAZ and G. BRANDUARDI-RAYMONT \\ Mullard Space Science Laboratory \\ University College London \\ Holmbury St. Mary, Dorking, Surrey \\ ENGLAND
}

We observed the Seyfert galaxy NGC6814 five times with EXOSAT between September 1983 and October 1985. For a more detailed description of the data see Mittaz and BranduardiRaymont (1988). Between observations both the LE $(0.02-2.5 \mathrm{keV})$ and ME $(2-6 \mathrm{keV})$ fluxes vary in the same sense, NGC6814 being brightest during observation 1 and weakest during observation 3 when it was undetected by the CMA. Both variable luminosity and variable low-energy absorption can explain this.

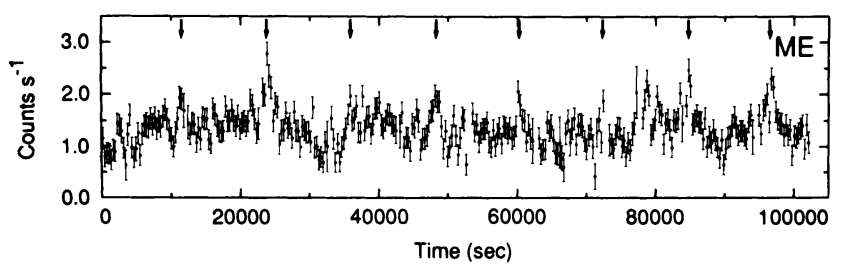

Figure 1. The timeline of the fifth observation with arrows showing the presence of small recurring flares.
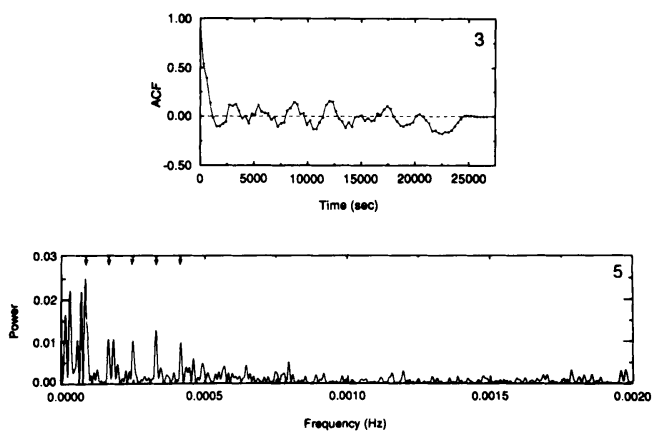

Figure 2. The autocorrelation function of the third observation and the Fourier transform of the fifth observation showing evidence of periodicities. The arrows indicate the fundamental frequency (at 12000s) and 4 harmonics. 
Within individual timelines variability is seen in the ME band (no variability could be detected in the LE due to poor statistics of the data). Small flares of approximately 1 hour duration are often seen. Uniquely, in observations 5 we find evidence for periodic behaviour both in the autocorrelation function and in the Fourier transform; in the latter peaks of a 12000 second period and 4 harmonics are clearly seen (Figure 2 bottom). This periodicity reflects the presence of small flares recurring at 12000 second intervals (Figure 1). In observation 3 the autocorrelation function shows a broad oscillation on a timescale of 3000 seconds (Figure 2 top). NGC6814 also displays fast variability. The most convincing rapid variation is seen in the ME flux in observation 2 where the flux drops by a factor of 2 or more from one 300 second bin to the next.

The hardness ratio (defined as the $4-6 \mathrm{keV} / 2-4 \mathrm{keV}$ ratio) is also variable but in a manner uncorrelated with the ME flux. The X-ray spectra themselves are all well fitted with a power law except for the first observation, where there is marginal evidence for a soft excess in the CMA with $\mathrm{Al} / \mathrm{Pa}$ filter at the $2 \sigma$ level. The spectra are found to vary both in slope and soft X-ray absorption, but in a fashion uncorrelated with the ME flux, in agreement with the behaviour of the hardness ratios.

One possible explanation of the unique flaring behaviour observed in observation 5 is that the recurrent flares are due to an instability in pair production processes in the plasma around a massive black hole at the nucleus of the galaxy (Moskalik and Sikora 1986). Reconstructing the flare shape from the harmonics in the Fourier transform, we find a similar shape to that predicted by the Moskalik and Sikora model (Figure 3). Using their model we calculate a black hole mass of $\lesssim 4 \times 10^{6} \mathrm{M}_{\odot}$. The spectral data support this model in that pair production may also explain the uncorrelated spectral variability.
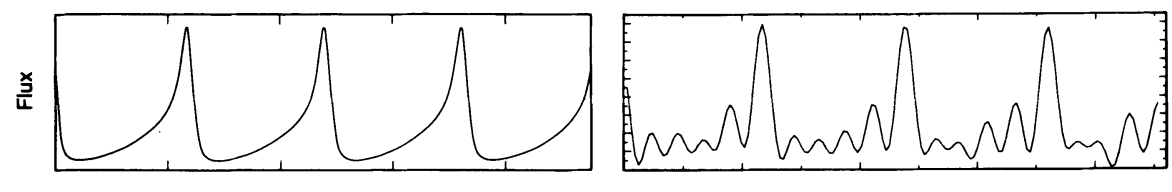

Time (arbitrary units)

Figure 3. The flare shape from the Moskalik and Sikora (1986) model (left panel) and the reconstructed flare shape of the fifth observation (right panel).

In conclusion, during two observations NGC6814 has been seen to exhibit periodic behaviour, in particular the recurring flares seen in observation 5 . This behaviour is unique to NGC6814. The flaring coupled with the characteristics of the spectral variability, leads us to the conclusion that pair production may be important in this object.

\section{References}

Mittaz, J.P.D. and Branduardi-Raymont, G. 1988, Mon. Not. R. astr. Soc., (submitted). Moskalik, P. and Sikora, M. 1986, Nature, 319, 649. 


\section{DISCUSSION}

LAWRENCE Have you calculated the probability of apparently observing such a periodicity by chance in a process that actually has a $l / f$ power spectrum?

MITTAZ No, I haven't calculated the probability from a $1 / f$ power spectrum, but I have calculated the probabilities of the peaks using the smoothed power spectrum of the data and adding in noise to create random data sets. I then found the number of times in 1000 such data sets a peak of the same significance as the peak of interest was found. In none of the random data sets was a peak found as significant as the 12000 second peak, and the harmonics were significant at over the $98 \%$ level.

BEALL 1. Could you comment on the uncertainty in the $300 \mathrm{sec}$ decrease in the NGC 6814 light curve? 2. I suggest that you (and all of us) adopt Schwartz's formalism as described in the $A p . J ., 318,568,1987$ to establish a consistent method for determining the minimum time scales of variability. Using that method, I believe the minimum time scale would be much larger than $300 \mathrm{sec}$.

MITTAZ 1. The timescale for the factor of 3 rapid decrease in flux is $280_{-100}^{+220}$ sec. 